\title{
Oxidative stress and nitric oxide synthesis in ISIAH rats with inherited stress-induced arterial hypertension
}

\author{
L. Klimov ${ }^{1,2 *}$, M. Zhuravina ${ }^{1,2}$ \\ ${ }^{1}$ Institute of Cytology and Genetics SB RAS, Novosibirsk, Russia \\ ${ }^{2}$ Novosibirsk State University, Novosibirsk, Russia \\ *e-mail:maple1708@mail.ru
}

Key words: ISIAH rats, hypertension, NO generation

Motivation and Aim: Hypertensive disease is widespread in modern society. It can be the cause of myocardial infarction, stroke and heart failure. One of the factors in the development of the disease is chronic emotional stress. The ISIAH rat strain (Inherited Stress-Induced Arterial Hypertension) is a rat model with the genetically determined enhanced responsiveness to stressful stimulation. The current population of the ISIAH rats is characterized by elevatedof both the basal arterial BP which reachesup to $175.0 \pm 3.5 \mathrm{mmHg}$ in males.

In addition to the four major systems responsible for BP control, there are number of local systems, in particular, the paracrine vasodilator - nitric oxide. Previously negative correlation of the bioavailability of nitric oxide in plasma and the level of BP in ISIAH rats was shown. The bioavailability of nitric oxide and its content in the kidneys and vessels is affected not only by the expression of the eNOS gene, but also by the concentration of reactive oxygen species (ROS) within the cell. The constitutive formation of ROS is necessary for the normal functioning of cells. Oxidative stress arises from an imbalance between antioxidant enzymes and enzymes that metabolize ROS, which leads to an increase in the formation of ROS. As a result, endothelium-dependent vasodilation and water-salt homeostasis can change, as ROS decreases the bioavailability of NO. Also previously it was shown a decrease in the activity of the antioxidant enzyme superoxide dismutase in blood plasma and a reduced concentration of the reduced form of glutathione in the blood plasma of ISIAH rats.

Methods and Algorithms: The relative amount of target mRNA was measured by qPCR. Lymphocytes were analyzed in 3-month old ISIAH and WAG rats (6 animals in each group). Lymphocytes were isolated from the blood plasma by centrifugation with LSM. Total RNA was extracted from lymphocytes and caudal arteries using the TRI reagent (Molecular research center, USA). Remaining traces of genomic DNA were removed from the RNA samples using DNase I (Promega, USA) treatment, according to the manufacturer's instructions. The value for the target gene was further normalized against the qPCR level of the reference gene.

Results: We found changes in the level of mRNA genes responsible for the synthesis of ROS in the lymphocytes and vessels of the ISIAH rats. Therefore we can assume a possible imbalance in the synthesis and metabolism of ROS, and as a result, the participation of oxidative stress mechanisms in the development of a stress-induced form of GB.

Acknowledgements: This work was supported by the Ministry of Education and Science of Russia (project No. 0324-2018-0016). 